\title{
ON THE EFFEGT OF SUBJECTIVE FACTORS UPON SIZE ESTIMATION
}

\author{
HIROTO KATORI
}

\section{University of Tokyo}

The experiment performed by Bruner and Goodman (2) seemed to indicate that the perceived size of an object was altered (accentuated) by its relevance to various needs of each individual. They found that although the sizes of neutral discs were estimated correctly, those of coins were overestimated in proportion to the monetary value, and the poorer children overestimated the coins to a greater extent than did the richer. In another experiment (4) Bruner and Postman had subjects estimate the size of discs bearing the dollar sign (as a positive valuedsymbol) and of discs bearing the Nazi emblem, the swastika (as a negative valued-symbol), and found that the former (positive) was judged larger than the latter (negative), though both were judged larger than discs bearing a neutral design.

These studies on the effect of " need" factors upon perception of size would be summarized to the following two propositions, i.e., (i) a " need "-related object, whether positive or negative, is overestimated than a non-" need "related object; (ii) the stronger need for an object an individual has, the more the size of the object is overestimated by him. Many experiments have been since then performed to test these propositions $(1,5,6,7,11,13,14,15)$. Some of them $(\mathbf{1}, \mathbf{5}, \mathbf{1 1}, \mathbf{1 4}, \mathbf{1 5})$ were confirmative, but some others $(\mathbf{6}, \mathbf{7})$ rather negative. In another (13) the effects of the "need" factors on the size estimations were certainly recog- nized, but in the contradictory direction to Bruner's proposition. I suppose, one of the causes of disagreements between these results would be owing to a methodological error which most of these experiments had commonly committed. That is to say, as Pastore said (12), if the "need"-related object were constructed by being added any symbol signifying any need character to the neutral one, we could not clearly decide whether what was effective on size estimation was any subjective variable other than the physical stimulus conditions or any change of physical stimulus conditions themselves, whatever shifts in estimated values might happen. Therefore, for the critical experiment, it is desirable to keep the physical qualities of stimulus to be estimated in the series of introducing subjective variables as same as in the series of estimating in neutral conditions. In the experiments by Lambert and others (8), as well as Lambert and Lambert (9), this point was taken into consideration: The subjective variables were operated in such ways as that previously neutral objects were connected with positive needs by conditioning and then disconnected by extinction. But some inefficiencies could be pointed out there in other points: (1) Their subjects were so young ( $3-5$ years old) that the tasks of the size estimations were almost impossible or very indefinite, especially for the youngest ; (2) their need operations were performed only on the posi- 
tive values.

The present author, considering these points, conducted a study in order to find, whether "need" factors, both positive and negative, are effecive or not on the size estimation of adults.

\section{EXPERIMENT 1}

\section{Method}

Subjects: Ninc male undergraduate students served as $S$ s. They were naive as to the purpose or procedure of the experiment.

Apparatus: The schematic representation of the apparatus is sketched in Fig. 1. $S$ sat at the intersection of the perpendicular lines of the two planes (Pl. A and Pl. B)

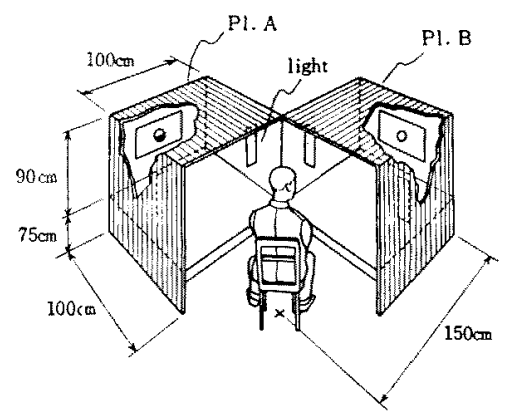

Fig. 1. The schematic representation of the apparatus.

on which a standard figure and a comparison figure were presented respectively; standard figures on $\mathrm{Pl} . \mathrm{A}$ and comparison figures on Pl. B. They were to be alternately observed in binocular vision.

The distance from $S$ to each plane was $1.5 \mathrm{~m}$. The left and the right fields were made equal in structure and in brightness (25 $\mathrm{ml}$; measured by photo-cell illuminometer). They were illuminated by four 40-watt tube-blubs with shadings by which $S$ 's eyes were protected from direct lights.

Figures: The standard figures, $50 \mathrm{~mm}$ in diameter, had one of the three kinds of designs: (A), (B) and (C) (Fig. 2). The comparison figures were outline circles, from $45 \mathrm{~mm}$ to $55 \mathrm{~mm}$ in diameter with

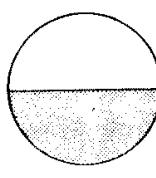

(A)

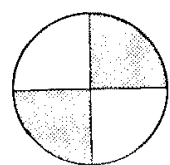

(B)

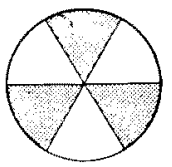

(C)
Fig. 2. Standard figures.

steps of $1 \mathrm{~mm}$. Each figure was drawn in black ink on a sheet of Kent paper ( $36 x$ $20 \mathrm{~cm})$; the width of lines drawn was about $0.8 \mathrm{~mm}$.

Method of measurement: Each estimated value was gained by the method of complete series. Judgments were made four times for each comparison stimulus, and three categories of judgments were employed.

Procedures: The experiment consisted of the following three sections: Pretest-section, Conditioning-section, and Extinctionsection. Each section was carricd out for three days respectively.

(i) Pretest-section

It had two parts; (1) " random presentation" of the standard figures, and (2) "size estimations" of the standard figures under the neutral conditions.

(1) After $S$ was first taught the names of the three standard figures which were called A, B and C respectively (see Fig. 2), standard figures were presented one by one in random order on $\mathrm{Pl}$. A, the names of which the $S$ was asked to call. This procedure was settled to make the stimulus situations in the pretest-section equal to those in the conditioning-section and to make $S$ familiar with the standard figures. Each standard figure was presented about fifteen times; the duration of a presentation was about $2-5 \mathrm{sec}$.

(2) Immediately after the above procedure, estimated size values of the three standard figures were measured one after another. The order of the standard figures presented for measuring had been designed for each $S$ (see Table 1); it was not changed 
for each $S$ throughout the all sections. The pretest-section, which consisted of (1) and (2), was repeated three times, one time par a day.

(ii) Conditioning-section

On the fourth day of the experiment, conditioning-section began. It had also two parts: (1) "conditioning" and (2) "size estimations". In the former part $S$ was exposed to a gambling situation in which he won or lost a sum of money and a set of symbolic meanings of standard figures was assigned to a $S$ according to the previously determined experimental design (see Table 1). $S$ was told, for example, "Now, we make a gambling. Figure $A$ has positive value for you and if figure $A$ is presented, you will win a bet. Figure $B$ has negative value, so you will lose one. Figure $\mathrm{C}$ has neutral value and neither you nor the experimenter win ".

The experimenter prepared such tables as illustrated in Fig. 3 for example, on which the names of three standard figures were arranged in three columns I-III and in fifteen series numbers 1-15. Each of the three standard figures appears in each series number once and in each column five times, in random order respectively. The experimenter showed $S$ one of the "tables" for a very short time, in order to dispel $S$ 's suspicion in the gambling where no artifices by the experimenter were inserted, and then instructed the method of gambling to $S$ as follows: $S$ had to declare one of the

\begin{tabular}{c|c|c|c}
\hline $\begin{array}{c}\text { Columns } \\
\text { Series } \\
\text { numbers! }\end{array}$ & I & II & III \\
\hline 1. & B & A & C \\
2. & A & C & B \\
3. & A & B & C \\
$\vdots$ & $\vdots$ & $\vdots$ & $\vdots$ \\
13. & B & A & C \\
14. & C & B & A \\
15. & A & C & B \\
\hline
\end{tabular}

Fig. 3. One of the "tables" used for the betting. three "columns", i.e., "I ", "II" or "III", at each betting of twenty yen. For example, when an $S$ at the first betting declard "III" and betted twenty yen, the figure $C$ which had been arranged on the first line of column III of the "table" in Fig. 3 was presented on $\mathrm{Pl}$. A. If figure $\mathrm{C}$, according to the preliminary agreement between the experimenter and the $S$, was neutral for the $S$, the twenty yen betted was returned to the $S$. In the second betting, if $S$ declared "I ", the experimenter had to present figure $\mathrm{A}$, which had been arranged on the second line of column I of the same "table". If A was the winning figure for the $S$, he should receive twenty yen. In the third betting, if $S$ declared "I" again, he won twenty yen. In every representations $S$ had to call the name of the standard figure, with its distinction of "positive", "negative", or "neutral", for example, "G-neutral " or "A-positive", etc. In such ways fifteen bettings were performed. $S$ was not allowed to declare the same "column" above five times successively. The duration of a presentation of each figure was about 2-5 sec.

Following this first part, size estimations of three figures were performed, as in the pretest-section. The order of measurement of standard figures was also same as in the pretest-section for each $S$. This section was repeated three times, one time par a day.

(iii) Extinction-section

This was entirely similar to pretestsection. On the seventh day of the experiment, $S$ was told that the gambles were over and he had to do the same task as on the pretest-section. It took also three days.

\section{Experimental Design}

The order of measurement of standard figures and the symbolic meanings of standard figures operated in the conditioning-section were counter-balanced by a kind of Latin square (see Table 1). 
Table 1

Experimental design

\begin{tabular}{c|c|c|c}
\hline $\begin{array}{c}\text { Order of } \\
\text { measurement } \rightarrow \\
\text { Subjects } \downarrow\end{array}$ & I & II & III \\
\hline Ak. & $($ A $)-(+)$ & $(\mathrm{B})-(-)$ & $(\mathrm{C})-(\mathrm{N}) *$ \\
Tk. & $(\mathrm{B})-(+)$ & $(\mathrm{C})-(-)$ & $(\mathrm{A})-(\mathrm{N})$ \\
Un. & $(\mathrm{C})-(+)$ & $(\mathrm{A})-(-)$ & $(\mathrm{B})-(\mathrm{N})$ \\
Yd. & $(\mathrm{A})-(-)$ & $(\mathrm{B})-(\mathrm{N})$ & $(\mathrm{C})-(+)$ \\
Kn. & $(\mathrm{B})-(-)$ & $(\mathrm{C})-(\mathrm{N})$ & $(\mathrm{A})-(+)$ \\
Kr. & $(\mathrm{C})-(-)$ & $(\mathrm{A})-(\mathrm{N})$ & $(\mathrm{B})-(+)$ \\
Oh. & $(\mathrm{A})-(\mathrm{N})$ & $(\mathrm{B})-(+)$ & $(\mathrm{C})-(-)$ \\
Kg. & $(\mathrm{B})-(\mathrm{N})$ & $(\mathrm{C})-(+)$ & $(\mathrm{A})-(-)$ \\
Ss. & (C)-(N) & $(\mathrm{A})-(+)$ & $(\mathrm{B})-(-)$
\end{tabular}

(A), (B) and (C) are the standard figures, $(+),(-)$ and $(\mathrm{N})$ are the "need" factors connected with the originally neutral standard figures in the conditioning-section: $(t)$, $(-)$ and $(\mathrm{N})$ stand for positive, negative and neutral, respectively.

\section{Results and Discussion}

The estimated values of the three standard figures, denoted by $(+),(--)$ or $(\mathcal{N})$, in every day by every $S$ are shown in Fig. 4. We see that: (1) Some $S$ s ( $S$. Un and $S$. Kr) showed rather fixed tendencies of overestimation and some others $(S . \mathrm{Yd}, S$. Oh, and $S$. Ss) those of underestimation from the actual size, irrespective of the "need" connotations of standard figures. (2) There are variations of the estimated values from day to day even in the same section. (3) Estimated values of positive and negative figures in the conditioning-section are not always larger than those either in the pretest-section or in the extinctionsection, or the diameter of the standard stimulus $(50 \mathrm{~mm})$. And even in the conditioning-section the positively or negatively valued-figures are not always overestimated than the neutral figures. Though the tendency of overestimation of both positive and negative

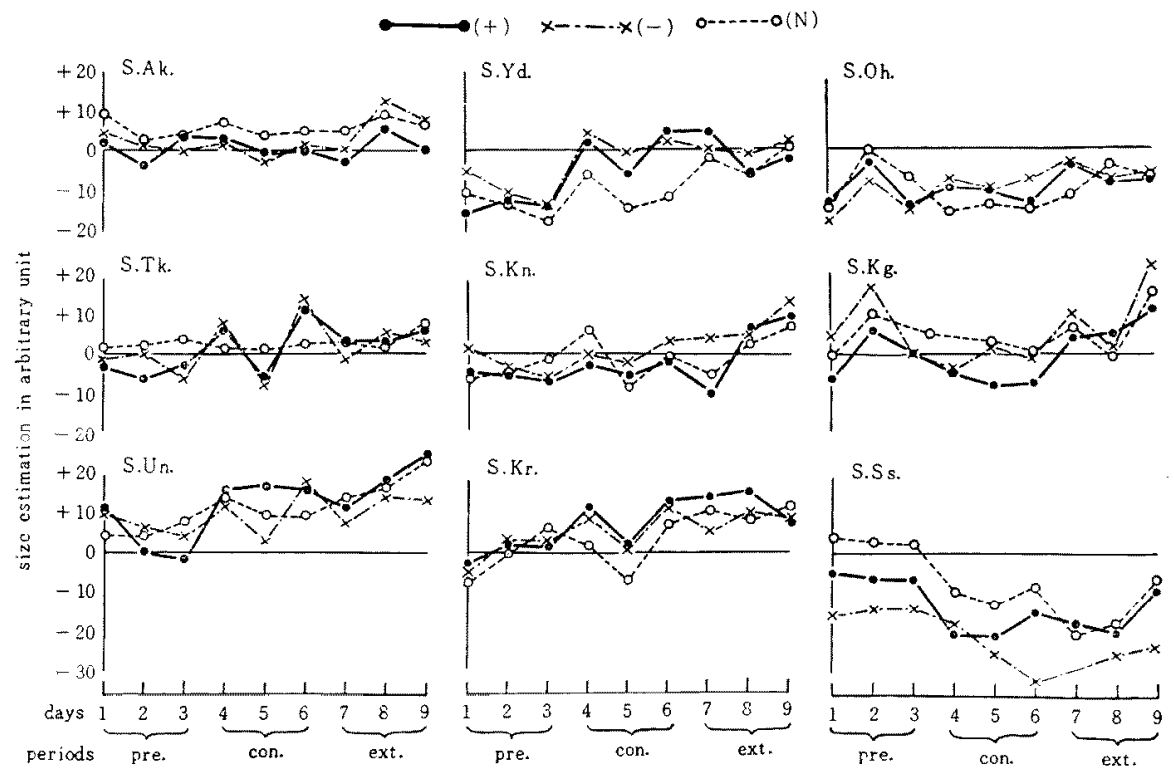

Fig. 4. Results of the size estimations of three standard figures by each $s$ in the three experimental sections. (Errors in size estimations are designated in an unit scale at which eight units are equivalent to $1 \mathrm{~mm}$. Zero level is set for the actual size $(50 \mathrm{~mm}$.). (+) represents overestimation and (-) understimation from the actual size. 
Table $2-\mathbf{a}$

\begin{tabular}{|c|c|c|c|c|c|c|c|c|c|c|}
\hline & \multicolumn{2}{|c|}{$(+) \sim(-)$} & \multicolumn{2}{|c|}{$(+) \sim(\mathbf{N})$} & \multicolumn{2}{|c|}{$(-) \sim(N)$} & \multicolumn{3}{|c|}{ Total } & \multirow[t]{2}{*}{$z^{2}$} \\
\hline & $<$ & $>$ & $<$ & $>$ & $<$ & $>$ & $<$ & $>$ & & \\
\hline Pretest & 16 & 11 & 12 & 15 & 14 & 13 & 42 & 39 & 81 & 1.19 \\
\hline Conditioning & 20 & 7 & 6 & 21 & 6 & 21 & 32 & 49 & 81 & $20.23 * *$ \\
\hline Extinction & 14 & 13 & 21 & 6 & 14 & 13 & 49 & 32 & 81 & 5.05 \\
\hline \multirow{2}{*}{ Total } & 50 & 31 & 39 & 42 & 34 & 47 & 123 & 120 & \multirow[b]{2}{*}{243} & \\
\hline & \multicolumn{2}{|c|}{81} & \multicolumn{2}{|c|}{81} & \multicolumn{2}{|c|}{81} & & & & \\
\hline
\end{tabular}

Table 2-b

\begin{tabular}{|c|c|c|c|c|c|c|c|c|c|c|}
\hline & \multicolumn{2}{|c|}{$(A) \sim(B)$} & \multicolumn{2}{|c|}{$(B) \sim(C)$} & \multicolumn{2}{|c|}{$(\mathrm{C}) \sim(\mathrm{A})$} & \multicolumn{3}{|c|}{ Total } & $z^{2}$ \\
\hline & $<$ & $>$ & $<$ & $>$ & $<$ & $>$ & $<$ & $>$ & & \\
\hline Pretest & 14 & 13 & 17 & 10 & 11 & 16 & 42 & 39 & 81 & 2.66 \\
\hline Conditioning & 11 & 16 & 9 & 18 & 12 & 15 & 32 & 49 & 81 & 0.73 \\
\hline Extinction & 18 & 9 & 16 & 11 & 15 & 12 & 49 & 32 & 81 & 0.73 \\
\hline \multirow{2}{*}{ Total } & 43 & 38 & 42 & 39 & 38 & 43 & 123 & 120 & \multirow[b]{2}{*}{243} & \\
\hline & \multicolumn{2}{|c|}{81} & \multicolumn{2}{|c|}{81} & \multicolumn{2}{|c|}{81} & & & & \\
\hline
\end{tabular}

Results of Experiment I: The frequencies of $<$ cases where the differences of size estimations of every two standard figures by each $S$ were less than $|0.5 \mathrm{~mm}|$, and $>$ cases where the above differences were more than $|0.5 \mathrm{~mm}|$.

$* * p<.01$.

figures than neutral figures is observed ( $S$. Yd, S. Kr and $S$. Oh), other tendencies, underestimation ( $S$. Ak and $S$. Ss) and alternation of overestimation and underestimation day by day (S. Tk), are also found. The three others ( $S$. Un, $S$. Kn and $S . \mathrm{Kg}$ ) do not show such definite tendencies. (4) These may be summarized to a more general tendency that, when a positive figure was overestimated in a day, a negative figures was almost always overestimated in the same day and vice versa. However, according to analysis of variance for estimated values of each $S$ in the conditioning-section (error: days $\times$ figures), only three $S \mathrm{~s}^{\prime}$ ( $S$. Ak,

* This was the arbitrary settled criterion, under which total cases were approximately divided into the same size groups (123:120).
S. Yd and $S . \mathrm{Kr}$ ) estimations were significant for the difference of the "need" comnotations at the level of $1 \%$.

To make this point more clear, Table 2 was arranged; in this table the frequencies of $<$ cases where the differences of estimated values of every two figures in the same day were less than $|0.5 \mathrm{~mm}|^{*}$ and $>$ cases where the above differences were more than $|0.5 \mathrm{~mm}|$, were listed; in Table 2-a on the differences between every two distinctions of the "need" connotations, irrespective of the figure designs. with which the "need" connotations were associated, i.e., $(+) \sim(-),(+) \sim$ $(\mathcal{N})$ and $(-) \sim(\mathcal{N})$, and in Table 2-b on the differences between every two figure designs irrespective of the "need" connotations, i.e., $(A) \sim(B),(B) \sim(C)$, 
and $(\mathrm{C}) \sim(\mathrm{A})$. Chi-squared tests were tried on each sections of Table 2-a and $2-b$ respectively.

Table 2-a shows that in the conditioning-section the frequencies of the $<$ cases in $(+) \sim(\mathcal{N})$ and $(-) \sim(\mathcal{N})$, are extremely less than those in $(+) \sim$ (-). Such remarkable deviations cannot be found in the pretest-section and the extinction-section of Table 2-a, and also anywhere in Table 2-b. Chisquared tests indicate also that there is significant relationship between the "need" connotations of figures and the differences of size estimations between any two figures in the Conditioning-section $\left(\chi_{0}^{2}=20.23>\chi_{0,01}^{2}, \quad d f=\right.$ $2)$; that is, in conditioning-section the figures relevant to " need", positive or negative, are distinguished in size estimation from the neutral figure, but positive figures and negative figures are not distinguished from each other in this respect. These tendencies were not found both in pretest-section ( $\chi_{0}^{2}=$ $\left.1.19<\chi_{0,05}^{2}, d f=2\right)$ and extinction-section $\left(\chi_{i=}^{*}=5.05<\chi_{i, 05}^{2}, \quad d f=2\right)$. We cannot find any statistical signification in the differences between every two figure designs (pretest-section: $\chi_{i}^{2}=$ $2.66<\chi_{0,05}^{2}$, conditioning-section : $\chi_{i 1}^{2}=$ $.73<\chi_{0,05}^{2}, \quad$ extinction-section: $\chi_{n}^{2}=.73$ $<\chi_{0,015}^{2}, d f=2$, respectively) .

\section{EXPERIMENT II}

One might be inclined to object the statement that the unexpected phenomena found in Experiment I would be only caused by the conditioning operation. The following experiment in which measurements of size were repeated nine times without the conditioning operation was conducted to answer this objections.

\section{Method}

Apparatus and procedures: The apparatus and stimulus figures were similar to Experi- ment I. The procedures were equal to the pretest-section of experiment $I$, except that (1) it was repeated nine times, one time a day, so that the conditioning-section and the extinction-section were ommited accordingly; and (2) the order of the three standard figures presented for the measuring was the same for all $S_{s}$ which was $(\mathrm{A}) \rightarrow(\mathrm{B}) \rightarrow(\mathrm{C})$. Four $S$ s were employed.

\section{Results and Discussion}

Table 3 indicates the frequencies tabulated under the same criterion, $0.5 \mathrm{~mm}$ as Table 2 in Experiment I. Nine experimental days were divided into three sections, corresponding to those in Experiment I.

From this table we find that:

The $<$ cases were more frequent than the $>$ cases, as compared with Experiment I and estimated values of every standard figures were less dispersing than those in Experiment I. The $p$-values in each section were derived from Fisher's exact treatment. No definite tendencies can be observed here in every section. Therefore, the above objection seems to be rejectable.

Our results in Experiment I and II seem to confirm the Bruner's first proposition, though a clear-cut tendency can not be found in every $S$, in so far as the "need "-related figures, both positive and negative, can be distinguished from the neutral figures in respect to the size perception, but do not support it over all, as the underestimation of the "need"-related figures appeared in some $S_{\mathrm{s}}$, which should not be explained by Bruner's term of "accentuation". Ohnishi also found that the positive objects were underestimated (11). We would not also regard our results as the phenomena of distortion, as it happened in our experiments that the constant errors in size estimations of the "need"-related figures from the actual 
Table 3

Results of Experiment II

\begin{tabular}{|c|c|c|c|c|c|c|c|c|c|c|}
\hline & \multicolumn{2}{|c|}{$(A) \sim(B)$} & \multicolumn{2}{|c|}{ (B) $\sim(\mathrm{C})$} & \multicolumn{2}{|c|}{$(\mathrm{C}) \sim(\mathrm{A})$} & \multicolumn{3}{|c|}{ Total } & \multirow[t]{2}{*}{$p$} \\
\hline & $<$ & $>$ & $<$ & $>$ & $<$ & $>$ & $<$ & $>$ & & \\
\hline 1 st -3 rd days & 9 & 3 & 7 & 5 & 6 & 6 & 22 & 14 & 36 & .074 \\
\hline 4th-6th days & 8 & 4 & 10 & 2 & 9 & 3 & 27 & 9 & 36 & .101 \\
\hline 7 th-9th days & 9 & 3 & 7 & 5 & 7 & 5 & 23 & 13 & 36 & .143 \\
\hline \multirow{2}{*}{ Total } & 26 & 10 & 24 & 12 & 22 & 14 & 72 & 36 & & \\
\hline & \multicolumn{2}{|c|}{36} & \multicolumn{2}{|c|}{36} & \multicolumn{2}{|c|}{36} & & & 108 & \\
\hline
\end{tabular}

size of standard figures $(50 \mathrm{~mm})$ were less than those in the neutral figures (see Fig. 4).

The terms as "schemata" of McCurdy would not immediately be applicable to our results as well as that of Lambert and others; because our "need "-related objects had been the neutral objects before they were subjected to the conditioning operation and had no relations with any kind of schemata which would be functioned in the experimental situations in the same direction as in daily situations.

So far the present results concern, we are unable to refer immediately to the Bruner's second proposition. The author, however, is in the opinion that this proposition is inconclusive, for the underestimations of positive figures happened fairly often and also the constant errors of neutral figures were not always less than those of "need"related figures. It becomes thus clear that any ready assumption or explanatory concept would be incompetent to explain our results completely.

So far as cognitive processes concern, the following may be tentatively discussed (cf. 3 Pp. 2-6) :

I) An organism ordinarily categorizes objects from their "functional " criterion. He then employs as cues, visual angle, brightness, shape and other physical ("formal") properties of objects which are common to these categories. Color patches distribution such as designs of figures used in our experiments, is also competent to be employed as a cue.

II) Through the conditioning operation in our experiment, stimulus figures come to obtain two opposed functional qualities (i.e., either "need". related category or neutral category). That is, the two standard figures which have been connected with positive or negative connotation in the gambling situation are classified into a single category, as " need "-related category, in the sense that these stimulus figures are somethings important to him. On the other hand, the standard figure which has been connected with neutral connotation is classified as neutral category, in the sense that the figure is something unimportant to him.

III) The cue of "need"-related category then would be just the opposite to that of neutral category. It would be possible, for example, that the cue of "need "-related category is disc area as having white sectors on blackground or vertical diameter of stimulus figures, on the one hand, and that of neutral category is disc area as having black sectors on white-ground or horizontal diameter of stimulus figures, on the other hand.

IV) In such situations of size 
comparison as our experiment, where the two stimulus objects compared are too apart presented each other to be viewed simultaneously, the size of standard figures may be estimated not by such cues as the visual angles subtended by the both ends of the stimulus figures, which would be the dominant otherwise, but by other cues available at that time (e.g., disc area or length of a diameter of disc). If a cue employed then would have the effect of over- or underestimation on size perception, a constant error (i.e., overor underestimation from the actual size) might result in the estimated value.

V) It is probable that the same cues as those of the functional categories would be also employed in the situation of size comparison as a "after-effect" of the categorical responses in the preceding conditioning operation. If the two cues transposed from the two functional categories in opposite relation have the antagonistic effects on size perception, the " need "-related figures, both positive and negative, may be distinguished from neutral figures as to size perception too. So far, when the cue of the "need"-related category happens to have the effect of the underestimation of size, both sizes of positive and negative figures might be possibly underestimated than those of neutral figures.

\section{EXPERIMENT III}

The above-mentioned discussion is only tentative. However, if it is confirmed that, as discussed in (IV), estimated value of the same standard figure is experimentally changeable by the difference of cues employed, the above hypotheses would become more plausible. Two experiments were conducted for this purpose. We took up, from various cues, disc area as having white sectors on black-ground or as having black sectors on white-ground (in Experiment III-a), as well as the vertical or the horizontal diameter of disc (in Experiment III-b).

\section{Method}

Apparatus and stimulus figures: The apparatus was similar to Experiment I and II. The visual fields were illuminated by a 350-watt flood-lamp behind $S(36 \mathrm{ml}$; measured by photo-cell illuminometer). The standard figures were equal to Experiment I and II (i.e., Figure A, B and C, $50 \mathrm{~mm}$ in diameter). In the series of the presentations of each standard figures two other figures which had the same kind of the designs as the standard figure, $48 \mathrm{~mm}$ and $52 \mathrm{~mm}$ in diameter respectively, were newly added and sometimes inserted randomly as a standard figure of "blank experiment" so as not to allow $S$ to get an inkling of the fact that the size of the standard figures were all equal. The comparison figures were outline circles from $43 \mathrm{~mm}$ to $57 \mathrm{~mm}$ in diameter with steps of $1 \mathrm{~mm}$.

\section{Procedures: (i) Experiment III-a......} Five graduate students served as $S$ s. Each $S$ was asked to estimate size of standard figures under employing such an appointed cue as either (a) disc area as having white sectors on black-ground, or (b) disc area as having black sectors on white-ground. The following instructions were given: "Please, estimate size of standard figures, directing your attention intensively to the white area, or to the black area of the standard figures and employing them alternately, according to the experimenter's direction ". The three standard figures, $50 \mathrm{~mm}$ in diameter, were presented to each $S$ under two cue-conditions (i.e., (a) the white area or (b) the black area), in random order. Six measurements of size estimation were accordingly assigned to each $S$, except for "blank experiments". The method of 
limits was used ; ascending series and descending series were repeated two times respectively.

(ii) Experiment III-b......Subjects were the same as in Experiment III-a. Each $S$ was asked to estimate size of the three standard figures under employing such a cue as (a) the vertical diameter, or (b) the horizontal diameter of the standard figure. Other procedures were all the same as in Experiment III-a.

Results and Discussion

Table 4 and 5 indicate estimated values of each $S$ for each standard figure under two cue-conditions in Experiment IIII-a and III-b respectively. The analysis of the data for both experiments is presented in Table 6 , from which we find that:

(1) In Experiment III-a, the $F$ for the cue-condition was not significant.
The $F$ for the individual differences $x$ the cue-conditions, however, was significant at better than the .05 level, besides the $F$ for the individual differences at better than .01 level. These mean that, though it can not generally be stated which of over- or underestimation would happen under employing which of cues of the white area or the black area, it is conceivable that in some $S$ s every standard figures were almost consistently judged larger in employing the cue of the white area than the cue of the black area, and in other $S$ s vice versa. (2) In Experiment III-b, not only the $F$ for the individual differences was significant at better than the .01 level, but also the $F$ for the cue-conditions at better than .05 level. That is, in every $S_{\mathrm{s}}$ standard figures were almost judged larger in employing the horizontal cue than the

Table 4

Estimated values (mm) in Experiment III-a ; cue-condition: white-black

\begin{tabular}{cccccccc}
\hline \multicolumn{2}{c}{$\begin{array}{c}\text { Figures } \\
\text { Cue-conditions } \rightarrow\end{array}$} & W & A & W & B & W & B \\
Subjects & & & & & & & \\
\hline Hr. & 50.1 & 50.5 & 48.6 & 50.1 & 50.4 & 52.0 \\
Ss. & 52.5 & 52.9 & 51.9 & 52.8 & 52.5 & 52.5 \\
Kk. & 50.5 & 50.4 & 48.9 & 50.1 & 46.3 & 49.5 \\
Kj. & 51.3 & 50.9 & 51.4 & 51.3 & 52.1 & 51.6 \\
Im. & 52.0 & 48.9 & 51.5 & 49.1 & 49.5 & 49.3
\end{tabular}

Table 5

Estimated values (mm) in Experiment III-b; cue-condition: horizontal-vertical

\begin{tabular}{|c|c|c|c|c|c|c|c|}
\hline \multirow{2}{*}{ Subjects } & \multirow{2}{*}{$\begin{array}{l}\text { Figures } \\
\text { Cue-conditions } \rightarrow\end{array}$} & \multicolumn{2}{|c|}{ A } & \multicolumn{2}{|c|}{$B$} & \multicolumn{2}{|c|}{$\mathrm{C}$} \\
\hline & & $\mathrm{H}$ & V & $\mathrm{H}$ & V & $\mathrm{H}$ & V \\
\hline & $\mathrm{Hr}$ & 49.4 & 48.8 & 50.0 & 49.0 & 49.8 & 49.4 \\
\hline & Ss. & 51.5 & 51.0 & 51.9 & 52.4 & 52.4 & 50.9 \\
\hline & Kk. & 52.3 & 51.1 & 52.0 & 49.8 & 51.0 & 49.5 \\
\hline & $\mathrm{Kj}$ & 51.3 & 51.9 & 52.3 & 51.3 & 52.0 & 51.1 \\
\hline & $\mathrm{Im}$ & 50.0 & 46.1 & 50.3 & 49.3 & 49.3 & 49.3 \\
\hline
\end{tabular}


Table 6

Summary of the results of the analysis of variance for each Experiment section

\begin{tabular}{|c|c|c|c|c|c|c|c|}
\hline \multirow{2}{*}{ Source } & \multicolumn{3}{|c|}{ Exp. III-a } & \multicolumn{3}{|c|}{ Exp. III-b } & \\
\hline & $d f$ & $M S$ & $F$ & $d f$ & $M S$ & $F$ & \\
\hline Cue-conditions & 1 & 85.87 & & 1 & 713.53 & $13.03^{*}$ & $F_{4}^{1}{ }_{21.20}^{717}$ \\
\hline Figures & 2 & 61.64 & & 2 & $64.9 \div$ & & $F_{g}^{2} \quad \begin{array}{l}4.40 \\
8.05\end{array}$ \\
\hline Individuals & 4 & 966.04 & $19.29 * *$ & 4 & 934.79 & $12.05^{* *}$ & $F_{8}^{4} \quad \begin{array}{l}3.84 \\
7.001\end{array}$ \\
\hline $\mathrm{C} \times \mathrm{F}$ & 2 & 86.37 & & 2 & 2.94 & & $F_{8}^{2} \quad \begin{array}{l}4.46 \\
8.85\end{array}$ \\
\hline $\mathrm{C} \times \mathrm{I}$ & 4 & 253.18 & $5.05 *$ & 4 & 54.78 & & 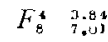 \\
\hline $\mathrm{F} \times \mathrm{I}$ & 8 & 135.38 & & 8 & 65.06 & & $F_{9}^{8} \begin{array}{ll}3 \cdot 44 \\
8 & 03\end{array}$ \\
\hline Residual & 8 & 50.09 & & 8 & 77.56 & & \\
\hline Total & 29 & & & 29 & & & \\
\hline
\end{tabular}

vertical cue.

From these results it may be concluded that the size estimation of the same standard figure is influenced differentially by the cues empoyed in such situations of size comparison as our experiments. It may be, moreover, stated that a prerequisite for the abovementioned hypotheses would be experimentally demonstrated hereby. Our results, therefore, suggest that the " need"-related figures, both positive and negative, may be distinguished from neutral figures as to size perception in the sense that the former are overestimated or underestimated than the latter, when (1) the cues employed in size estimation happen to be the same as those of the functional categories of these figures in other situations and (2) the cue of "need "-related category is just the opposite (e.g., whitefigure on black-ground or vertical diameter) to that of neutral category (e.g., black-figure on whiteground or horizontal diameter) and (3) the two groups of cues result the antagonistic effects on size perception (e.g., one has the effect of overestimation and another has that of underestimation).

\section{SUMmary}

Three experiments were conducted to test the assumption that "need"-related objects, either positive or negative, would be accentuated than neutral objects. In Experiment I, a kind of a conditioning was employed, that is, three originally neutral figures were connected with monetary values, i.e., positive, negative or neutral connotation, in a gambling situation. After this procedure $S$ was asked to estimate the size of these figures. The experiment was conducted for nine days. The results of this experiment showed that "need"-related figures, positive and negative, tended to be distinguished from neutral figures, but both positive and negative were either overestimated or underestimated than neutral figures. In Experiment II it was found that these facts could not happen without the conditioning operation. Experiment III showed that the size estimation of the same stimulus figure was influenced by the difference of the cues employed. It was discussed that these 
results might be explained in terms of "cue difference" by a after-effect of the categorical responses in the conditioning operation, rather than in terms of "accentuation ", "schemata" etc.

\section{REFERENCES}

1. Ashley, W.R., Harper, R. S., \& Runyon, D. L. The perceived size of coins in normal and hypnotically induced economic states. Amer. F. Psschol., 1951, 64, $564-572$.

2. Bruner, J. S., \& Goodman, C. C. Value and need as organizing factors in perception. 7. abnorm. soc. Psychol., 1947, 42, 33-44.

3. Bruner, J. S., Goodnow, J. J., \& Austin, G.A. A study of thinking. New York: Wiley, 1956.

4. Bruner, J. S., \& Postman, L. Symbolic value as an organizing factor in perception. J. soc. Psychol., 1948, 27, 203208.

5. Brtner, J. S, \& Rodrigues, J. S. Some determinants of apparent size. $\mathcal{F}$. abnorm. soc. Psychol., 1953, 48, 17-24.

6. Carter, L.F., \& Schooler, K. Value, need, and other factors in perception. J. exp. Psychol., 1949, 56, 200-207.

7. Klein, G. S., Schlesinger, H. J., \& Meister, D. E. The effect of personal values on perception: An experimental critique. Psychol. Rei., 1951, 58, 96112.

8. Lambert, W. W., Solomon, R. L., \& Watson, P. D. Reinforcement and extinction as factors in size estimation. 7. exp. Psychol., 1949, 35, 637-641.

9. Lambert, W.W., \& Lambert, E.C. Some indirect effects of reward on children's size estimations. F. abnorm. soc. Psychol., $1953,48,508-510$.

10. MCCurdy, H. G. Coin perception studies and the concept of schemata. Psychol. Roi., 1956, 63, 160-168.

11. Ohnishi, K. Note: Perceived size of "valued object". Jinbun-kenkyu, 1954, 5, 76-86.

12. Pastore, N. Need as a determinant of perception. F. Psychol., 1949, 28, 457475.

13. Rosenthal, B.G., \& Levy, J.H. Value, need and attitude toward money as determinant of perception. Amer. Psychologist, 1950, 5, 313. (Abstract)

14. Solly, C.M., \& Lee, R. Perceived size: Closure versus symbolic value. Amer. 7. Psychol., 1955, 68, 142-14t.

15. Vroom, V.H. Design and estimated size of coins. Canat. F. Pṣychol., 1957, 11, 89-92.

(Receiced March 30. 1959) 